\title{
The Coalition of Cancer Cooperative Groups
}

\section{Robert L. Comis, $M D$}

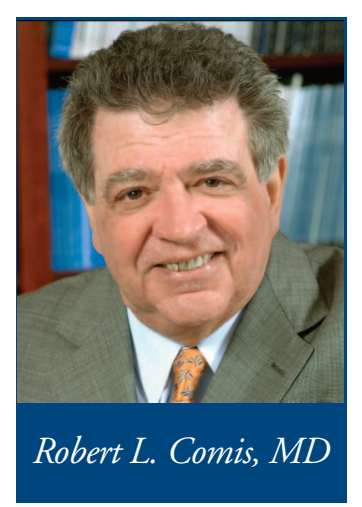

and provides information about cancer clinical trials available throughout the country. In partnership with IMPAC Medical Systems Inc, TrialCheck is the only clinical trials database integrated into an electronic medical record system.

In 2005, the Coalition and Northwestern University, Chicago, Illinois, developed a web-based survey of attitudes and awareness of cancer survivors to investigate the reasons behind low levels of patient participation in clinical trials. The results of this survey were recently presented at the 43rd Annual Meeting of ASCO, Chicago, Illinois, in a poster presentation. ${ }^{4}$ The survey found that the majority of cancer survivors would have considered enrolling in a clinical trial if their physician had made them aware of the option. The findings underscore that physician communication and attitudes about cancer clinical trials are pivotal to patient awareness and enrollment.

A nonprofit organization founded in 1997, the Coalition's mission is to improve the quality of life and survival of cancer patients by increasing participation in cancer clinical trials. The Coalition has created and made available a suite of programs and services designed to increase the understanding of cancer clinical trials and boost participation. Since the founding of the Coalition in 1997, patient participation in Cooperative Group trials has gone up by about $30 \%$.

The Cooperative Groups are the country's clinical research engine, treating more than one half of all patients participating in clinical trials in the United States. This year the Cancer Cooperative Groups became the first group to receive an American Society of Clinical Oncology (ASCO) Distinguished Service Award for Scientific Leadership, recognizing the "team science" achievements of the Cooperative Groups in designing and conducting cancer clinical trials.

The Coalition has worked closely with ASCO in fostering clinical trials participation in community-based practices. Since 2003, the Coalition has been the sole sponsor of the ASCO Clinical Trials Participation Award, which has recognized 53 excellent community-based practices. Also, it cosponsors, along with ASCO and the Oncology Nursing Society, the ASCO Clinical Trials Workshop where teams of physicians and nurses are trained and educated about best practices for successful clinical trials sites.

Building national awareness and educating the public about clinical trials is the focal point of the Coalition's National Awareness Campaign. The award-winning Knowledge is Power, an 8-page patient education supplement in Newsweek magazine, is the program centerpiece. ${ }^{1} \mathrm{It}$, along with other educational material for patients, is available on the Coalition Web site. ${ }^{2}$

Another Coalition program geared toward increasing patient accrual is TrialCheck. The TrialCheck database ${ }^{3}$ is updated daily

\section{References}

1. Knowledge is Power: A Patient's Guide to Cancer Clinical Trials! Newsweek, June 2, 2003

2. Coalition of Cancer Cooperative Groups: Welcome to CancerTrialshelp.org. www.CancerTrialsHelp.org

3. Trial Check: Welcome to TrialCheck.org.www.TrialCheck.org

Recently, in the area of support and funding for cancer research, the Coalition was at the forefront of an effort to halt a $10 \%$ decrease in NCI funding of the Cooperative Groups, which would have come on top of a steady decrease in NCI funding since 2002. The issue was covered in the Wall Street Journal and the USAToday, among other media outlets.5,6 On May 22, 2007, the Cooperative Groups received notice from the NCI that program budgets for fiscal year 2007 will remain stable at the fiscal year 2006 level. However, ultimately, a reversal of the downward trend and an increase in funding is needed to keep pace with the rising costs of clinical research and the increasing number of new agents to investigate.

Throughout the past decade, the Coalition has maintained and refined its focus on its mission. However, more work needs to be done, especially in the important area of bridging government, physicians, industry, and patient advocates. The Coalition's Scientific Leadership Councils are designed to bring national leaders in research and the cancer community together to address significant gaps in knowledge, unanswered scientific questions, and unfulfilled medical needs that potentially delay or jeopardize long-term prospects for developing and implementing new approaches to research. The task of the Councils is to recommend and profile the most promising research in specific diseases, and convene stakeholders in the cancer community to help form common goals and understanding around these research needs.

Robert Comis, MD, is President and Chairman of the Coalition of Cancer Cooperative Groups, is Professor of Medicine and Director of the Drexel University Clinical Trials Research Center, Philadelphia, Pennsylvania, and the Group Chair of the Eastern Cooperative Oncology Group.

4. Comis RL, Colazzi DD, Miller JD: Barriers to cancer clinical trials (CCT) participation: "We have met the enemy and he is us." J Clin Oncol 25:338s, 2007 (abstr 6567) 5. Funding Concerns Hit Some Cancer Trials. February 07, 2007. The Wall Street Journal 6. Proposal would cut cancer institute budget again. USA Today, January 29, 2007. http:/www.cancertrialshelp.org/pdfs/usa.pdf 